\title{
RSSI-based Indoor Tracking using the Extended Kalman Filter and Circularly Polarized Antennas
}

\author{
Moez Ben Kilani, Alexandre J. Raymond, François Gagnon and Ghyslain Gagnon \\ Laboratoire de communications et d'intégration de la microélectronique (LACIME) \\ École de technologie supérieure \\ Montreal, Canada \\ moez.ben-kilani.1@ens.etsmtl.ca, \\ alexandre.raymond@lacime.etsmtl.ca, \\ \{francois.gagnon,ghyslain.gagnon\}@etsmtl.ca
}

\author{
Philippe Lavoie \\ Quattriuum Inc. \\ Quebec, Canada \\ p.lavoie@quattriuum.com
}

\begin{abstract}
A tracking scenario comprising a mobile emitter node moving through an indoor environment covered by multiple anchor receivers is investigated in this work. A localization method based on received signal strength indicators (RSSI) and making use of the extended Kalman filter (EKF) and circularly polarized (CP) antennas is proposed. The EKF implements the position-velocity (PV) model, which assumes that the target is moving at a near-constant velocity during any given short time interval $\Delta t$. The measurement vector is composed of velocities in addition to RSSI values, which allow to deal with the error term between measurements and the propagation model directly. CP antennas are used on both the anchor nodes and the mobile node. These antennas are known to reduce the effects of multipath, especially those caused by single reflections. As a result, the RSSI values received in line of sight are more accurate and stable than those received from linearly polarized antennas. We tested our approach by tracking the movement of a robot following a predefined trajectory. The maximum location estimation error (LEE) is found to be $0.52 \mathrm{~m}$. In addition, velocity changes are easily tracked during the target movement, which demonstrates the effectiveness of the proposed approach.
\end{abstract}

Keywords: indoor localization, RSSI, EKF, CP antennas.

\section{INTRODUCTION}

Wireless indoor positioning systems have received a lot of attention in recent years. Various types of wireless sensor networks have been investigated for different types of sensors (radio-frequency, infrared, optical, inertial, etc.) and estimation algorithms [1]. For radio-frequency-based systems, several signal metrics, such as time of flight measurements (TOA, TDOA) and direction of arrival measurements (AoA), were detailed in [2], [3]. Systems based on received signal strength indicators (RSSI) have also been studied extensively, as they have a comparatively low cost and can leverage pre-existing infrastructure, such as Wi-Fi and Bluetooth networks [4].

However, the RSSI-based indoor positioning technique is highly dependent on the propagation environment, which can lead to significant localization errors. For instance, permanent changes in the physical environment can yield inaccuracies with respect to the propagation model. Those permanent changes can be caused by multipath fading due to signal reflections, which is problematic in RSSI-based localization systems.

In order to mitigate those issues, many improvements were proposed [1]. The first type of improvements is mainly algorithmic. In fact, different estimators were investigated in order to enhance the positioning accuracy.

In [4], [5], a least square (LS) estimator was proposed for RSSI-based location estimation. This linear estimator attempts to minimize the error term between measurements and propagation model. An adaptive approach was proposed in [5], taking into account the dynamic changes in the propagation environment. Specifically, a joint estimation technique of unknown location coordinates and path-loss exponent was investigated. However, in order to apply the non-linear LS algorithm, a linearization step based on the first-order Taylor series expansion and the Levenberg-Marquardt method is required, which leads to additional complexity.

A maximum likelihood (ML) based estimator was detailed in [6]. The proposed approach also dynamically estimates the propagation parameters, based on real-time RSSI measurements. The main drawback of such approach is again the amount of calculations needed to perform the algorithm.

A second type of location estimation improvements were investigated in [7]-[9]. Those improvements involve the fusion of RSSI measurements with data from other types of sensors (inertial, laser, etc.). This approach was shown to clearly increase the accuracy of the proposed localization techniques.

The Kalman filter (KF) is one of the well-known predictioncorrection algorithms. It can be easily adapted to tracking scenarios [10], [11]. However, since RSSI measurements relate to physical coordinates in a non-linear fashion, the extended Kalman filter (EKF) is more suitable, as it applies some linearizations and approximations around the current estimate using the partial derivatives of the process and measurement functions [11], [12]. In [13], adaptive approaches using the EKF with direct RSSI measurements were proposed, and better results were obtained compared to more traditional LS estimators. 
Recently, circularly polarized (CP) antennas have shown much promise in mitigating the effects of multipath fading in indoor environments [14], [15]. Such antennas also allow a more flexible reciprocal orientation of the transmitter and the receiver. As such, they are becoming widely used in several wireless applications, such as the global positioning system (GPS), synthetic aperture radar (SAR), as well as radiofrequency identification systems (RFID) [14].

Circular polarization was also shown to reduce the rootmean-square delay spread by about one-half compared to linear polarization (LP), and the bit error rate (BER) due to multipath propagation in high-speed transmission channels [16], [17]. In [14], circular polarization was applied to an RSSI-based localization system. A direct comparison between measured and estimated position based on a standard Hatalike model was proposed for both LP and CP antennas. It was clearly shown that lower localization errors were obtained using CP antennas. In our work, we extend the study investigated in [14] by proposing a solution to reduce the estimation errors in tracking scenarios.

This paper aims to design and evaluate the accuracy of a simple and robust algorithm based on the EKF estimator, suitable for indoor tracking of mobile nodes. The proposed algorithm processes raw RSSI measurements directly, taken from wireless receivers equipped with $\mathrm{CP}$ antennas. The combination of the CP antennas, which yield more stable RSSI values, and the EKF, which offers excellent tracking performance, is evaluated. To the best of the authors' knowledge, it is the first time CP antennas are combined with an EKF estimator to perform RSSI-based indoor localization.

The remainder of this paper is organized as follows: Section II presents the tracking approach based on the extended Kalman filter and CP antennas. Section III describes the experimental setup used to validate our proposed technique, and presents performances results. Finally, Section IV concludes this paper.

\section{SYSTEM COMPONENTS}

This section describes each component of our proposed localization system.

\section{A. Extended Kalman Filter}

As mentioned in the previous section, our tracking approach is based on an extended Kalman filter, operating in the discrete time domain. This filter recursively estimates the state of a dynamic system modeled by the following state equation [13]:

$$
\mathbf{X}_{k}=f\left(\mathbf{X}_{k-1}\right)+\mathbf{w}_{k},
$$

where $\mathbf{X}_{k}$ is the state vector at time $k, f(\cdot)$ is the state transition function which projects a state vector $\mathbf{X}_{k-1}$ forward in time, and $\mathbf{w}_{k} \sim \mathcal{N}\left(0, \mathbf{Q}_{k}\right)$ is a random variable modeling random process noise, normally distributed with zero mean and covariance matrix $\mathbf{Q}_{k}$.

We use the position-velocity model [13] to characterize the state vector $\mathbf{X}$, which is defined as:

$$
\mathbf{X}=\left[\begin{array}{llll}
x & y & v_{x} & v_{y}
\end{array}\right]^{T},
$$

where $x$ and $y$ are the coordinates of the node on a twodimensional plane, and $v_{x}$ and $v_{y}$ are the corresponding velocities along those axes, respectively. The node's height is assumed constant in this model.

We also assume that the mobile node moves with a constant velocity between adjacent time intervals. Any change in target velocity is modeled as acceleration noise included in $\mathbf{w}_{k}$; it also models non-linearities and system perturbations.

The transition function models a constant-speed, linear motion:

$$
f\left(\mathbf{X}_{k-1}\right)=\mathbf{F}_{k} \mathbf{X}_{k-1}=\left[\begin{array}{cccc}
1 & 0 & \Delta t & 0 \\
0 & 1 & 0 & \Delta t \\
0 & 0 & 1 & 0 \\
0 & 0 & 0 & 1
\end{array}\right] \mathbf{X}_{k-1}
$$

where $\Delta \mathrm{t}$ is the short time interval during which the mobile node velocity is assumed constant. The value of $\Delta \mathrm{t}$ is chosen to be $0.1 \mathrm{~s}$ due to hardware limitations.

$\mathbf{Q}_{k}$ is computed assuming the acceleration as a white noise random vector. This assumption takes into consideration different forces that could temporally cause changes in target directions as described in [18] :

$$
\mathbf{Q}_{k}=a^{2}\left[\begin{array}{cccc}
\frac{\Delta t^{3}}{3} & 0 & \frac{\Delta t^{2}}{2} & 0 \\
0 & \frac{\Delta t^{3}}{3} & 0 & \frac{\Delta t^{2}}{2} \\
\frac{\Delta t^{2}}{2} & 0 & \Delta t & 0 \\
0 & \frac{\Delta t^{2}}{2} & 0 & \Delta t
\end{array}\right]
$$

where $a$ is the maximum amplitude of the process noise.

The measurements are considered during the update phase. They are incorporated into the filter using:

$$
\mathbf{Z}_{k}=h\left(\mathbf{X}_{k}\right)+\mathbf{v}_{k}
$$

where $\mathbf{v}_{k} \sim \mathcal{N}\left(0, \mathbf{R}_{k}\right), \mathbf{Z}_{k}$ is the measurement vector at instant $k$, and $h(\cdot)$ is the observation function which estimates the expected measurements at the true state $\mathbf{X}_{k} \cdot \mathbf{v}_{k}$ is the measurement noise vector, modeled as a normally distributed random variable with zero mean and covariance matrix $\mathbf{R}_{k}$, which we set to a diagonal matrix as we assume that the measurements errors are independent.

In order to take advantage of the non-linear capabilities of the EKF, the system makes direct use of the RSSI measurements $P_{\text {ref }}$ produced by the receivers in the observation vector $\mathbf{Z}$, in addition to the measured velocities:

$$
\mathbf{Z}=\left[\begin{array}{llllll}
v_{x} & v_{y} & P_{\operatorname{ref}_{1}} & \ldots & P_{\operatorname{ref}_{L}}
\end{array}\right]^{T} .
$$

The measurements $v_{x}$ and $v_{y}$ contained in the measurement vector gives additional information regarding the node's state, which the algorithm uses to improve tracking accuracy. Inaccuracies related to the measured velocities are also taken into account through the measurement noise covariance matrix:

$$
\mathbf{R}_{k}=\operatorname{diag}\left(\sigma_{v_{x}}^{2} \sigma_{v_{y}}^{2} \sigma_{\mathrm{dBm}_{\mathrm{ref}_{1, k}}^{2}}^{2} \ldots \sigma_{\mathrm{dBm}_{\mathrm{ref}_{L, k}}^{2}}^{2}\right) .
$$

Matrix $\mathbf{R}_{k}$ characterizes the errors between measured and propagation-model-based RSSI values. More stable RSSI measurements allow us to have smaller error variances, thus the 


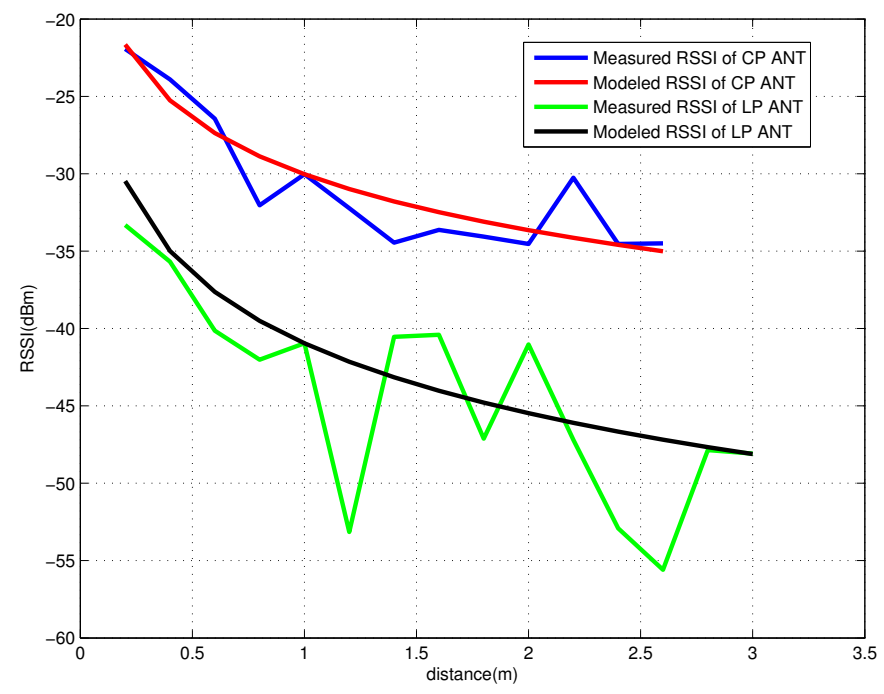

Fig. 1: Experimental and modeled propagation characteristics of CP and LP antennas

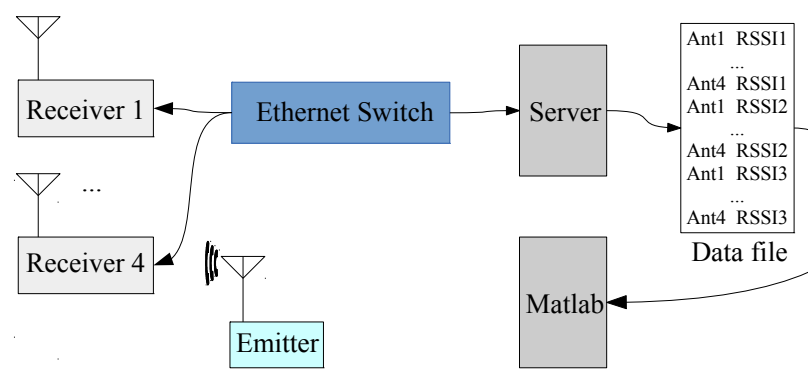

Fig. 2: High-level description of the acquisition system

EKF trusts actual RSSI measurements more than the predicted ones. Good tracking performance can therefore be achieved provided that the RSSI measurements are accurate.

The observation function is derived from the log-normal propagation model applied to each receiver:

$$
h\left(\mathbf{X}_{k}\right)=\left[\begin{array}{c}
v_{x} \\
v_{y} \\
P_{0_{1}}-10 \alpha_{1} \log _{10}\left(\operatorname{dist}\left(\mathbf{X}_{k}, \mathbf{X}_{\mathrm{ref}_{1}}\right) / d_{0}\right) \\
\vdots \\
P_{0_{L}}-10 \alpha_{L} \log _{10}\left(\operatorname{dist}\left(\mathbf{X}_{k}, \mathbf{X}_{\mathrm{ref}_{L}}\right) / d_{0}\right)
\end{array}\right],
$$

where $\alpha_{i}$ is the path loss exponent related to receiver $i, L$ is the number of receivers, $P_{0_{i}}$ is the mean power received at a distance $d_{0}$ (typically $1 \mathrm{~m}$ ) from the receiver $i, \mathbf{X}_{\text {ref }_{i}}$ is the position of the receiver $i$, and $\operatorname{dist}(\cdot)$ is the Euclidean distance function.

\section{B. CP Antennas}

Compared to LP antennas, CP antennas offer better performances by reducing multipath effects, which yields more stable RSSI measurements. Those characteristics reduce estimation errors, especially those due to first-order signal reflections. Indeed, when a circularly polarized wave is reflected,

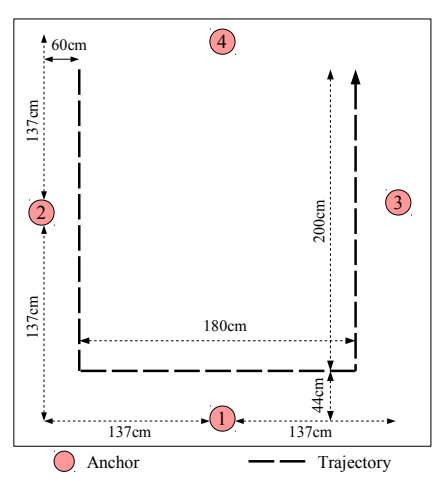

(a) Anchor positions

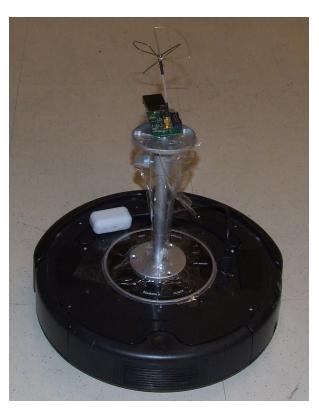

(b) Robot and emitter
Fig. 3: Experimental configuration

its handedness is reversed. Thus, if the transmitting and receiving antennas are circularly polarized with the same handedness (both right-handed or left-handed CP), multipathdelayed waves caused by single reflections will be effectively rejected by the receiving antennas. This characteristic is of great interest because channel fading is generally caused by first-order reflections, and because the field amplitude of such reflections is much higher than those of higher-order reflections [14], [15].

In order to characterize the advantages of using $\mathrm{CP}$ antennas compared to LP antennas, we carried out RSSI measurements using both types. Those results are presented in Figure 1. High RSSI fluctuations are obtained for the LP case, due to the superposition of incident and reflected waves, resulting in constructive and destructive interference. Conversely, reduced oscillations can be observed when CP antennas are used, as expected, due to the reduced amplitude of the first-order reflections. The RSSI error-term variance $\sigma_{\mathrm{dBm}}^{2}$ was found to be $19.87 \mathrm{dBm}^{2}$ for LP antenna, compared to $7.73 \mathrm{dBm}^{2}$ for $\mathrm{CP}$ one. Propagation parameters were determined in both cases ensuring minimum error term variances between the measurements and the propagation model. The same transmitted power was used for both experiments. Note that the higher values of RSSI obtained with the CP antenna is related to its higher gain compared to the LP one.

\section{EXPERIMENTATION}

In order to test the performance of the proposed system, a localization experiment was devised.

\section{A. Experimental Setup}

Our experimental setup consists of four sensor nodes, or anchor nodes, positioned outside a capture area of $4 \mathrm{~m} \times$ $4 \mathrm{~m}$, as illustrated in Figure 3a. Both the transmitter and the receivers are equipped with circularly polarized, omnidirectional antennas operating at $2.4 \mathrm{GHz}$.

Experiments were carried out using custom-built receivers based on Texas Instruments CC2510 $2.4 \mathrm{GHz}$ radio transceivers [19], and equipped with the four-leaf receiver antennas shown in Figure 4a. Those receivers are connected, 


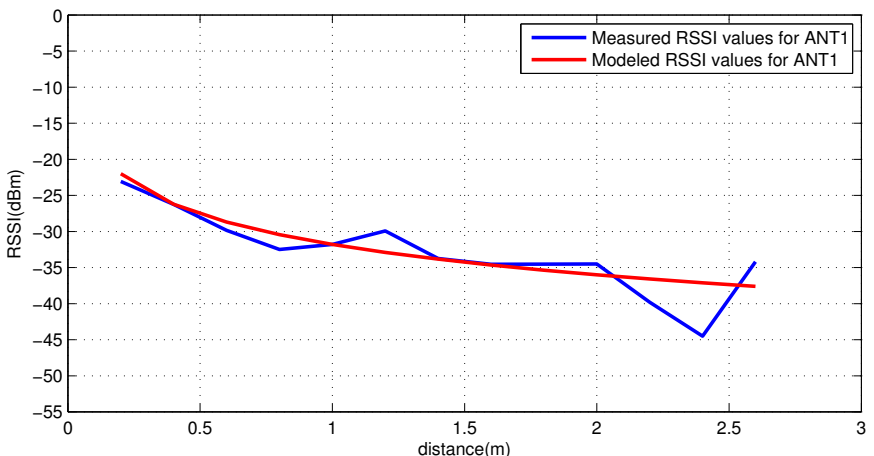

(a) Receiver 1

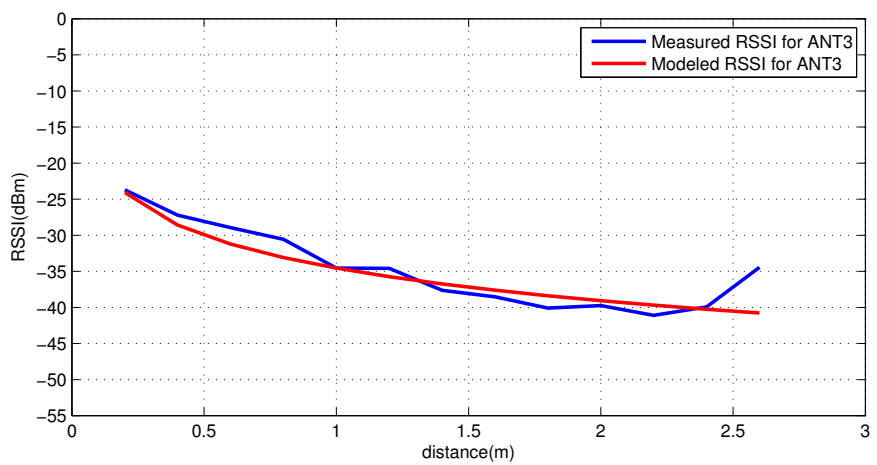

(c) Receiver 3

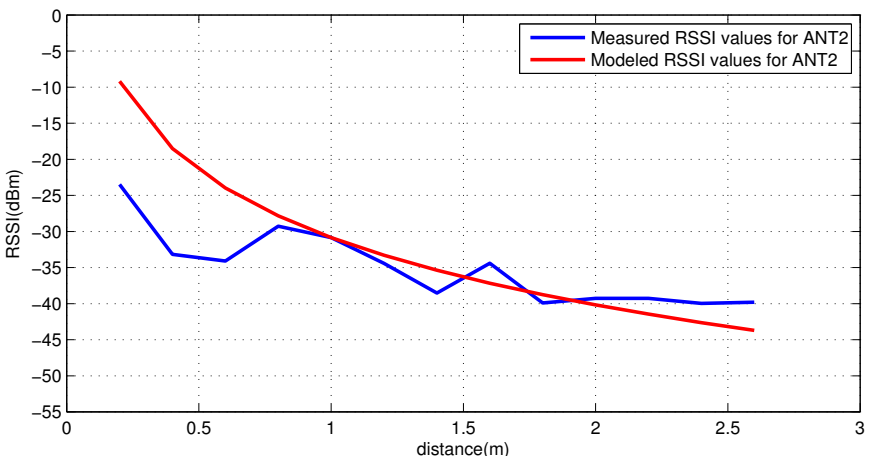

(b) Receiver 2

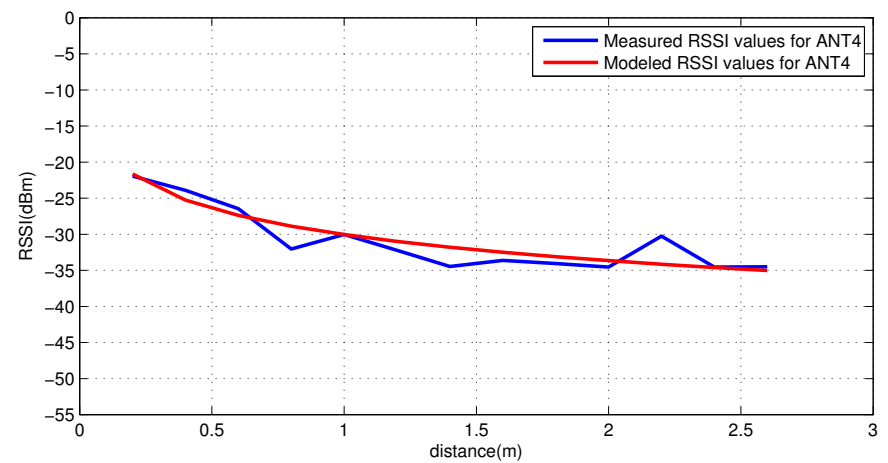

(d) Receiver 4

Fig. 5: Log-normal channel model of different receivers

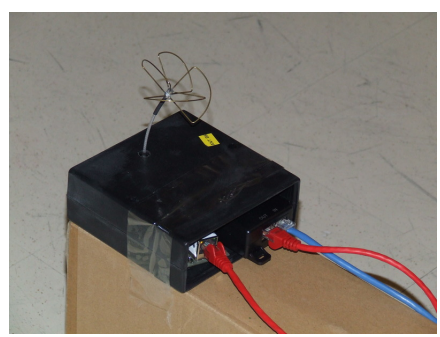

(a) Receiver (anchor nodes)

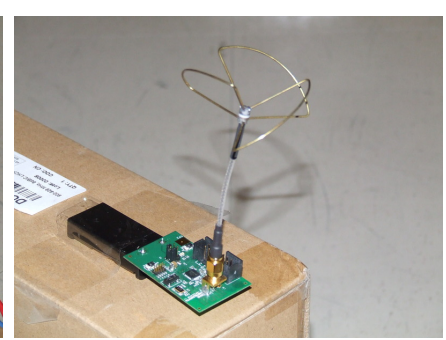

(b) Emitter
Fig. 4: Custom-built receiver and emitter, equipped with circularly polarized $2.4 \mathrm{GHz}$ antennas

via ethernet links, to a central processing server where data is saved for offline processing.

The emitter also consists of a CC2510 module, programmed to permanently transmit a sinusoidal signal on an unused channel of the $2.4 \mathrm{GHz}$ ISM band. The mobile node makes use of a three-leaf transmitter antenna, as presented in Figure 4b. We installed the emitter on top of an iRobot Roomba robot, depicted in Figure $3 \mathrm{~b}$, programmed to follow a piecewiselinear trajectory at a constant speed of $0.2 \mathrm{~m} / \mathrm{s}$. This trajectory is illustrated in Figure 3a.

Due to the presence of Wi-Fi signals in the same frequency band, we were careful to choose an unused channel so as to limit the amount of interference.

Figure 2 describes the acquisition system from a high- level point of view. The four receivers are connected to a computer server via an ethernet link. To reduce the number of wires required, the receivers are powered using a powerover-ethernet-compatible ethernet switch. The computer server gathers RSSI measurements from each receiver at $100 \mathrm{~ms}$ intervals. Those measurements are stored in a plain-text file for offline processing using MATLAB.

\section{B. Calibration Phase}

TABLE I: Propagation parameters

\begin{tabular}{|c|c|c|c|}
\hline Antenna $i$ & $P_{0_{i}}(\mathrm{dBm})$ & $\alpha_{i}$ & $\sigma_{\mathrm{dBm}_{\mathrm{ref}_{i}}}^{2}$ \\
\hline 1 & -31.79 & 1.4 & 7.32 \\
\hline 2 & -30.84 & 3.1 & 7.73 \\
\hline 3 & -34.55 & 1.5 & 4.79 \\
\hline 4 & -30.035 & 1.2 & 3.06 \\
\hline
\end{tabular}

In order to model the propagation characteristics of the receiver antennas, an offline calibration phase was first carried out. 100 RSSI measurements were recorded after placing the emitter at various distances from each receiver, in $20 \mathrm{~cm}$ increments.

Those received values were then imported into MATLAB for analysis, and the parameters of each antenna were computed by minimizing the error term variance $\sigma_{\mathrm{dBm}_{\text {ref }_{i}}}^{2}$, assuming a log-normal propagation model. The resulting model parameters are illustrated in Figure 5. The mean received power 
at distance $d_{0}=1 \mathrm{~m}$, path-loss exponent $\alpha_{i}$ of the adjusted model, and the error-term variances are summarized in Table I.

\section{Localization Accuracy}

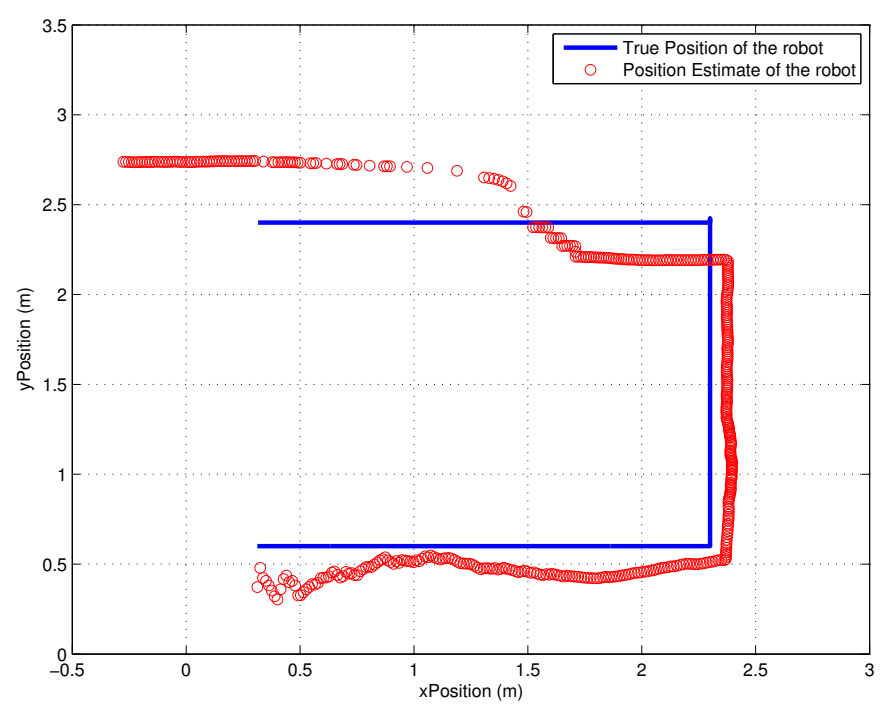

Fig. 6: Position tracking performance

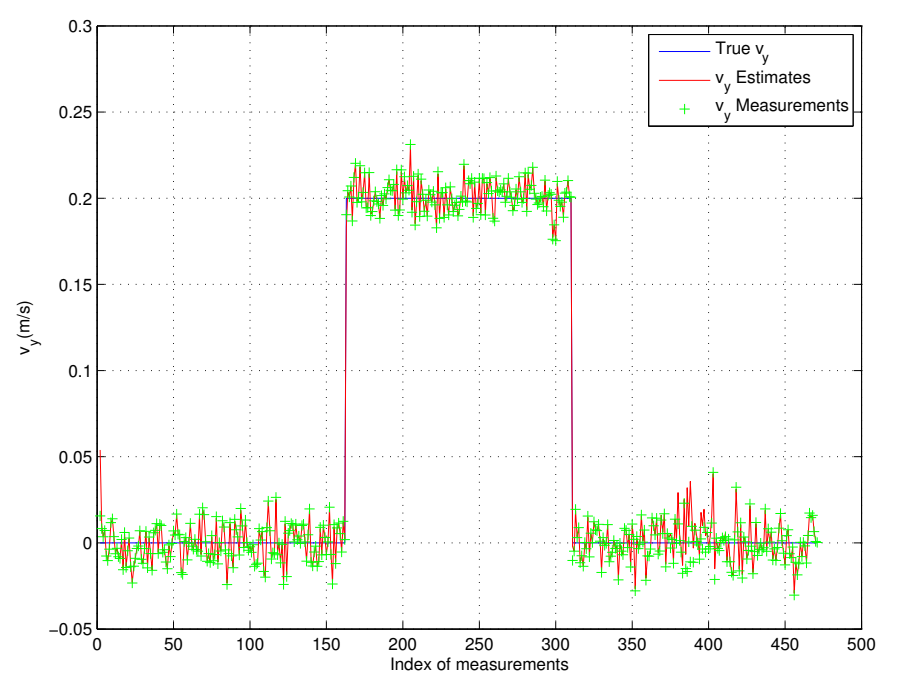

Fig. 7: Velocity tracking performance in the y direction

After this initial calibration step, which characterized the antennas as well as the propagation environment, we carried out a tracking scenario to evaluate the precision of the proposed system quantitatively.

The mobile node was moved along the trajectory presented in Figure 3a. RSSI measurements were gathered from all four receivers and sent to the computer server. A total of 471 measurements were gathered from each receiver. These measurements were then processed using the EKF implemented in MATLAB code.

After processing, the target's estimated trajectory was computed and compared to a known ground truth. Comparative results are shown in Figure 6. The maximum location estimation error (LEE) is found to be $0.52 \mathrm{~m}$. We can see from the estimation curve that changes in direction are also well predicted by the EKF.

The tracking precision is essentially dependant on the stability of measurements obtained from $\mathrm{CP}$ antennas, as detailed in previous sections, as well as the additional noisy velocity measurements supplied to the algorithm. This latter point is corroborated by Figure 7, which demonstrates the effectiveness of the proposed EKF model in estimating the y-axis velocity during the tracking scenario. It also illustrates the fluctuations of $v_{y}$ caused by changes in target direction along its path. The additional information regarding measured velocities allow us to increase the tracking ability of the proposed system, and to avoid the imprecisions introduced by the simplistic PV model.

\section{CONCLUSION}

A cost-effective and easily-deployable tracking approach based on the use of circularly-polarized antennas and the extended Kalman filter is presented in this paper.

RSSI values gathered from four deployed CP receivers allow us to track a mobile node using the EKF. RSSI measurements are used directly in the EKF observation model, improving the performance of the filter.

Although a small number of anchor receivers was used, the maximum radial estimation error is found to be $0.52 \mathrm{~m}$. This allows us to consider this architecture as a candidate for indoor localization purposes in more complex indoor environments, although additional CP receivers will likely be required.

Future work will focus on implementing a frequency diversity scheme, as well as the addition of other sensor technologies, such as inertial measurements.

\section{ACKNOWLEDGEMENT}

The authors want to thank Quattriuum Inc for their financial support and technical assistance during the project.

This work was sponsored by the Natural Sciences and Engineering Research Council of Canada (NSERC).

Thanks are due to ReSMiQ for their partial support to this project.

\section{REFERENCES}

[1] A. Fink and H. Beikirch, "Analysis of rss-based location estimation techniques in fading environments," in Indoor Positioning and Indoor Navigation (IPIN), 2011 International Conference on, 2011, pp. 1-6.

[2] M. Kanaan and K. Pahlavan, "A comparison of wireless geolocation algorithms in the indoor environment," in Wireless Communications and Networking Conference, 2004. WCNC. 2004 IEEE, 2004, pp. 177-182.

[3] H. Liu, H. Darabi, P. Banerjee, and J. Liu, "Survey of wireless indoor positioning techniques and systems," Systems, Man, and Cybernetics, Part C: Applications and Reviews, IEEE Transactions on, vol. 37, no. 6 , pp. 1067-1080, 2007.

[4] H. Laitinen, S. Juurakko, T. Lahti, R. Korhonen, and J. Lahteenmaki, "Experimental evaluation of location methods based on signal-strength measurements," Vehicular Technology, IEEE Transactions on, vol. 56, no. 1, pp. 287-296, 2007.

[5] X. Li, "Rss-based location estimation with unknown pathloss model," Wireless Communications, IEEE Transactions on, vol. 5, no. 12, pp 3626-3633, 2006 
[6] S. Mazuelas, A. Bahillo, R. M. Lorenzo, P. Fernandez, F. A. Lago, E. Garcia, J. Blas, and E. J. Abril, "Robust indoor positioning provided by real-time rssi values in unmodified wlan networks," Selected Topics in Signal Processing, IEEE Journal of, vol. 3, no. 5, pp. 821-831, 2009.

[7] A. Fink, H. Beikirch, M. Voss, and C. Schroder, "Rssi-based indoor positioning using diversity and inertial navigation," in Indoor Positioning and Indoor Navigation (IPIN), 2010 International Conference on, 2010, pp. 1-7.

[8] W.-W. Kao and S.-H. Lin, "Dead-reckoning aided rssi based positioning system for dynamic indoor environments," in Proceedings of the $23 \mathrm{rd}$ International Technical Meeting of The Satellite Division of the Institute of Navigation (ION GNSS 2010), 2010, pp. 3359-3365.

[9] J. Schmid, T. Gadeke, W. Stork, and K. Müller-Glaser, "On the fusion of inertial data for signal strength localization," in Positioning Navigation and Communication (WPNC), 2011 8th Workshop on, 2011, pp. 7-12.

[10] R. Kalman, "A new approach to linear filtering and prediction problems," Journal of Basic Engineering, vol. 82, no. 1, pp. 35-45, 1960.

[11] G. F. Welch and G. Bishop, "An introduction to the kalman filter," University of North Carolina, Chapel Hill, NC, USA, Tech. Rep, 1995.

[12] J. Yim, C. Park, J. Joo, and S. Jeong, "Extended kalman filter for wireless lan based indoor positioning," Decision Support Systems, vol. 45, pp. 960-971, 2008.

[13] M. A. Caceres, F. Sottile, and M. A. Spirito, "Adaptive location tracking by kalman filter in wireless sensor networks," in Wireless and Mobile Computing, Networking and Communications, 2009. WIMOB 2009. IEEE International Conference on, 2009, pp. 123-128.
[14] P. Nepa, F. Cavallo, M. Bonaccorsi, M. Aquilano, M. C. Carrozza, and P. Dario, "Experimental analysis of rssi-based indoor location systems with wlan circularly polarized antennas," Wireless Mobile Communication and Healthcare Lecture Notes of the Institute for Computer Sciences, Social Informatics and Telecommunications Engineering, vol. 55, pp. 176-183, 2011.

[15] R. Szumny, K. Kurek, and J. Modelski, "Attenuation of multipath components using directional antennas and circular polarization for indoor wireless positioning systems," in Radar Conference, 2007. EuRAD 2007. European, 2007, pp. 401-404.

[16] T. S. Rappaport and D. A. Hawbaker, "Wide-band microwave propagation parameters using circular and linear polarized antennas for indoor wireless channels," Communications, IEEE Transactions on, vol. 40, no. 2, pp. 240-245, 1992.

[17] T. Manabe, K. Sato, H. Masuzawa, K. Taira, T. Ihara, Y. Kasashima, and K. Yamaki, "Polarization dependence of multipath propagation and highspeed transmission characteristics of indoor millimeter-wave channel at 60 ghz," Vehicular Technology, IEEE Transactions on, vol. 44, no. 2, pp. 268-274, 1995.

[18] M. Kohler, "Using the kalman filter to track human interactive motion modelling and initialization of the kalman filter for translational motion," Technical Report 629, University of Dortmund, Germany, 1997.

[19] Wireless Connectivity Proprietary 2.4 GHz CC2510F32, Texas Instruments, available online: http://www.ti.com/products/cc2510f32. 